Abstract 375 Table 1 Table of investigations

\begin{tabular}{|c|c|c|c|c|c|c|}
\hline & $\begin{array}{l}\text { Prior } \\
\text { chemotherapy }\end{array}$ & $\begin{array}{l}\text { D2 post } \\
\text { Chemotherapy }\end{array}$ & $\begin{array}{l}\text { D3 post } \\
\text { chemotherapy }\end{array}$ & $\begin{array}{l}\text { D6 post } \\
\text { chemotherapy }\end{array}$ & $\begin{array}{l}\text { D11 post } \\
\text { chemotherapy }\end{array}$ & $\begin{array}{l}\text { D19 post } \\
\text { Chemo } \\
\text { therapy }\end{array}$ \\
\hline Plaletet & 33 & 7 & 42 & 142 & & 152 \\
\hline AST & 40 & 5381 & 7978 & 938 & 100 & 79 \\
\hline ALT & 16.4 & 2035 & 3524 & 1829 & 610 & 127 \\
\hline Bilirubin & 4.9 & 29.6 & 49.9 & 34.5 & 37.8 & 19.9 \\
\hline Ammonia & & 125 & 84 & & & \\
\hline PT & & 33.7 & 34.5 & 18.8 & & \\
\hline
\end{tabular}

brain revealed no intracranial bleed and ultrasound abdomen showed gross ascites. Multidisciplinary teams discussion led to diagnosis of HTS.

She was closely monitored in intensive care unit with vigorous supportive care included high flow nasal cannula, judicious fluid and urine output monitoring.

She showed tremendous improvement, regained full GCS within 24 hours of admission. Her platelet count had marked increment by second day of admission and normalized within one week. All the blood parameters showed improvement within a week.

She received subsequent chemotherapy with reduction of actinomycin D dose with no recurrence of HTS.

Results The onset of HTS occurred within 10 weeks of diagnosis and usually occurred 1 to 7 days post actinomycin D administration. HTS can last up to 12 days and resolve with supportive treatment. After recovery, most children can tolerate actinomycin $\mathrm{D}$ at a reduction dosage without recurrence of the syndrome.

Therapy of HTS is mainly vigorous supportive care. However, defibrotide, which is an antithrombotic has emerged as an effective therapy in view of histopathologic marker of HTS is obliteration of hepatic vein.

Conclusions Early detection and recognition of syndrome will prompt immediate action resulting extremely favourable outcome. Further studies regarding risk factors and promising drugs should be carried out to reduce mortality of HTS.

\section{POSTERIOR REVERSIBLE ENCEPHALOPATHY SYNDROME IN ACUTE POST-STREPTOCOCCAL GLOMERULONEPHRITIS}

Nurhidayah Zulkifli, Siew Sing Chua, Lay Chin Tan, Fong Chiao Lai, Noraini Ab Rahman, Chin Hong Ding. Malaysia

\subsection{6/bmjpo-2021-RCPCH.206}

Background Acute post streptococcal glomerulonephritis (PSGN) is well known sequel of group A $\beta$-hemolytic streptococcus infections and may be complicated with hypertensive encephalopathy followed by Posterior reversible encephalopathy syndrome (PRES). PRES is also known as acute hypertension encephalopathy, usually present with seizures, hypertension, headache, encephalopathy and/or visual disturbance. The pathophysiology behind PRES is poorly understood. It is usually associated with hypertensive encephalopathy, kidney disease, autoimmune disease, immunosuppressive drug and organ transplantation. Contrast-enhanced computed tomography imaging allows for fast assessment which shows features of edema of the posterior cerebral hemisphere, especially at the parieto-occipital region. PRES can be a presenting sign of PSGN and vice versa.

Objectives To study relationship between PSGN and PRES

Methods Two case reports PSGN with PRES, based on contrast-enhanced computed tomography (CECT) brain.

Results We reported two cases of PSGN with PRES.

First case is a 11 years old boy, who presented with status epilepticus, following one-day history of headache and vomiting. He was afebrile, no history of epilepsy or recent trauma and any drug intake. He had history of left facial cellulitis secondary to odontogenic infection one-week prior the onset of seizure. He was initially diagnosed with PRES after CECT brain.

Second case is a 6 years old boy, who presented with multiple infected impetigo lesions over bilateral lower limb, fever and vomiting. He also developed blurry vision and recurrent vomiting, requiring ICU care due to hypertensive crisis. CECT brain was performed and reported as PRES.

Similarly, both children had persistent microscopic hematuria and hypertension, and investigations show low C3 and normal C4 levels with raised Anti-streptolysin O titer (ASOT), fulfilling the criteria of PSGN.

Conclusions The incidence of PRES is rare in children, but should always be a diagnosis to be considered in children with PSGN presenting with hypertensive crisis and/or rapidly progressive neurological manifestations, e.g. status epilepticus. Early recognition and prompt treatment are important measures in reducing morbidity and mortality.

\section{THINK OUT OF THE BOX! LYMPHADENOPATHY, SKIN RASHES AND SKULL LESIONS; ALL ENDED UP WITH LANGERHANS CELL HISTIOCYTOSIS ?}

Lay Chin Tan, Siew Sing Chua, Fong Chiao Lai, Norsarwany Mohammad, Chin Hong Ding, Noraini ab rahman. Malaysia

\subsection{6/bmjpo-2021-RCPCH.207}

Background Langerhans cell histiocytosis (LCH), previously known as histiocytosis $\mathrm{X}$, is a rare paediatric disorder. The abnormal proliferation of specialized dendritic cells found in skin and mucosa, which resembles langerhans cells, account for the underlying pathology of $\mathrm{LCH}$ (1). The vast clinical presentations of $\mathrm{LCH}$, from self-limiting lesion to fulminant disseminated disease, make its diagnosis a challenge for doctors. Its incidence stands at around 1-5 per million children. Some literatures showed that children of parents who were exposed to certain chemicals such as benzene have an 
increased risk for developing LCH. We presented 3 cases, 2 of whom aged 6 months and below.

Objectives To provide a wider perspective in diagnosing $\mathrm{LCH}$. Methods We are presenting 3 case reports, 2 of whom aged 6 months and below.

Results 1st case: Inguinal swelling mimicking inguinal abscess

This was a case report of a 6-month-old girl who presented with multiple lymphadenopathies over the right inguinal, axillary and cervical region. She was otherwise asymptomatic. A detailed history revealed presence of the right inguinal swelling noted since 2 weeks old and progressively increasing in size. Detailed investigation for infection were all normal. She received few courses of antibiotics but symptoms persisted. The father works in road construction industry. Excision biopsy of the right inguinal lymph node showed positive staining of CD1a, confirming the diagnosis of LCH.

2nd case: Skin rashes mimicking eczematous rashes

This was a 5-month-old girl who was admitted for fever with petechiae rashes, while the blood investigations revealed pancytopenia. 3 weeks prior to admission, she developed skin rashes mimicking eczema over the genitalia and bilateral inguinal. Systemic examinations showed hepatosplenomegaly, ascites with multiple petechiae rashes over the groin and neck region. Full blood picture reported pancytopenia with leucoerythroblastic blood film and circulating blasts. Her mother works in company selling household cleaning detergent. Skin biopsy of the inguinal region confirmed the diagnosis of $\mathrm{LCH}$.

3rd case: Scalp swelling mimicking scalp abscess

This was a 2-year-5-month old 'Orang Asli' (native people), whose stays in rubber estate in a remote area (regular exposure to pesticides), presented with multiple scalp swellings. Other systemic examination was unremarkable. Blood investigations and septic parameters were normal. Computed tomography (CT) brain showed multiple soft tissue mass with osteolytic bony destruction, involving outer and inner table of occipital, left parietal and left frontal bone. Excision biopsy of the scalp confirmed the diagnosis of LCH.

Conclusions LCH has a wide spectrum of clinical manifestations. Although it is a very rare disease, LCH should always be considered as differential diagnosis in children with generalised lymphadenopathy, scalp swelling or any disorder with multisystemic organs involvement. A thorough history to identify risk, physical examination and laboratory investigation are vital to aid in diagnosing patient.

\section{MATERNAL AND INFANT PREDICTORS OF GROWTH TRAJECTORIES IN SINGAPORE CHILDREN IN THE FIRST 18 MONTHS OF LIFE}

Tultul Das, Truls Ostbye, Yoke Hwee Chan, Ngiap Chuan Tan, Elaine Chew. Singapore

\subsection{6/bmjpo-2021-RCPCH.208}

Background Childhood obesity, with overweight defined as BMI $z$-score $>2$ based on WHO standards, is a growing problem in Singapore. The proportion of overweight children aged 6-18 years in Singapore has increased from 11\% in 2013 to $13 \%$ in 2017. This prevalence persists into childhood as $70 \%$ of children who were overweight when 7 years old remained overweight at 11 years of age. Rapid weight gain during infancy is an established risk factor for later obesity and excess body weight tracks from infancy into childhood and adulthood. However, little is known about the growth trajectories of infants in Singapore.

Objectives The objective of this study was to examine the longitudinal growth trajectories of infants in Singapore by tracking weight gain and Body Mass Index (BMI) (both absolute and BMI z-scores) in the first 18 months of life. Additionally, maternal and child predictors of infant weight and BMI were examined. Maternal predictors include number of months of breastfeeding, maternal age, and maternal education level while infant predictors include ethnicity and gender.

Methods Retrospective data on infants attending a government-subsidised primary care clinic between July 2019 to August 2020 was collected. Maternal characteristics (maternal age at birth and maternal education level at the child's 18month visit) and infant characteristics (birth weight, birth height, infant gender and infant ethnicity) were recorded from electronic medical records. Weight and BMI were measured at birth, 1, 3, 6, 9, 12 and 18 months. The type of feeding (breastfeeding or formula feeding or soft diet or a mix of any of the 3) was reported at each visit. Mixed Model Repeated Measures analysis was performed to compare weight and BMI within each gender, ethnicity and number of months of breastfeeding.

Results 7125 infants attended the clinic during the study period. $50.5 \%$ of the participants were male. The majority of infants were Chinese at $68.7 \%, 22.8 \%$ were Malay and $5.1 \%$ were Indian. We observed a peak in BMI at 6 months of age, with a mean of $17.5 \mathrm{~kg} / \mathrm{m}^{2}$. BMI then declined thereafter to $16.9 \mathrm{~kg} / \mathrm{m}^{2}$ at 12 months. We identified gender and ethnic differences in weight and BMI trajectories. Males had a higher mean weight and BMI than females across all time points. Chinese infants generally had a higher mean weight and BMI as compared to Malay and Indian infants. Indian infants exhibited the lowest BMI across all time points. With regards to feeding, $86.8 \%$ of the children were breastfed at 1 month of age and the prevalence declined to $54.8 \%$ by 6 months. Breastfeeding duration was identified as an important predictor of weight and BMI as a longer duration of breastfeeding was associated with a lower weight and BMI. Longer duration of breastfeeding was associated with a lower weight and BMI even when compared to those within the same gender and race.

Conclusions This study shows that gender, ethnicity and breastfeeding duration are early life predictors of rapid weight gain. Importantly, breastfeeding is a potentially modifiable predictor that may form the basis of targeted prevention efforts to reduce the risk of obesity.

\section{BONE DENSITY IN ASIAN ADOLESCENTS WITH ANOREXIA NERVOSA AND ATYPICAL ANOREXIA NERVOSA}

Eric Tay, Chu Shan Elaine Chew. Singapore

\subsection{6/bmjpo-2021-RCPCH.209}

Background Bone Mineral Density is frequently measured during the assessment and management of adolescents with Eating Disorders (EDs) such as Anorexia Nervosa (AN) and Atypical AN(AAN), which negatively affects the physical and mental developments of children at a vital phase of growth. Compromised bone accrual in adolescents has significant long-term implications on their peak bone mass and overall bone health 